\title{
Teatro para convivir: investigación-acción para el desarrollo de habilidades sociales en jóvenes costarricenses con trastorno del espectro autista
}

\author{
Theater to live together: action-research for the development of social \\ skills in young Costa Ricans with autism spectrum disorder
}

\section{Palabras clave}

Trastorno del espectro autista, teatro, tecnología, habilidades sociales, educación especial

\section{Keywords}

Autism spectrum disorder, theatre, technology, social skills, special education

\author{
Linda Madriz Bermúdez \\ <lmadriz@uned.ac.cr> \\ Universidad Estatal a Distancia. \\ Costa Rica
}

\section{Alicia Sandoval Poveda <asandovalp@uned.ac.cr> \\ Universidad Estatal a Distancia. Costa Rica}

\section{Viviana González Rojas <vgonzalez@uned.ac.cr>}

Universidad Estatal a Distancia. Costa Rica

\section{Paloma Antón Ares}

<palomanton@edu.ucm.es>

Universidad Complutense de Madrid. España

ción social y la interacción en diferentes contextos (deficiencias de reciprocidad socioemocional, conductuales, comunicativas no verbales, en el desarrollo, en el mantenimiento y comprensión de las relaciones) así como patrones restrictivos de comportamiento, intereses o actividades (movimientos repetitivos, insistencia en la monotonía, inflexibilidad de rutinas, intereses restringidos y fijos anormales en intensidad o foco de interés, hiper o hipoactividad a los estímulos sensoriales). En este patrón se incluye a las personas con un diagnóstico previo de una condición de ásperger o autismo.

La detección del TEA suele producirse en la infancia, etapa en la que se centran la mayoría de los estudios. Los síntomas comportamentales varían a lo largo del crecimiento, pero no desaparecen del todo. Esta variación hace necesario realizar un abordaje con las personas que tienen esta condición, a lo largo de la vida, no solo en la primera infancia (Cuesta et al., 2016; Riesgo et al., 2013; Martos-Pérez y Llorente-Comí, 2013; Organización Mundial de la Salud, 2013). Un momento vital de cambio es el final de la adolescencia, cuando aquellos jóvenes que han estado insertos en el sistema educativo culminan su proceso de

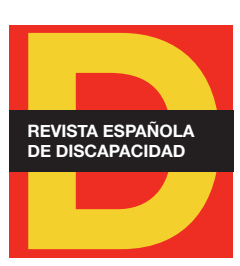

Para citar:

Madriz, L. et al. (2019): "Teatro para convivir: investigación-acción para el desarrollo de habilidades sociales en jóvenes costarricenses con trastorno del espectro autista". Revista Española de Discapacidad, 7 (II): 165-183.

Doi: <https://doi.org/10.5569/23405104.07.02.10> 
formación académica en educación secundaria, aunado a las condiciones propias de esta etapa de la vida que generan crisis por los cambios. No todos logran insertarse después de este periodo en estudios técnicos, superiores o en un entorno laboral, y sus posibilidades de recreación e interacción con pares se ve limitada al no encontrarse en el mismo contexto que estos.

Debido a su condición, una persona con TEA puede tener dificultades para alcanzar oportunidades de empleo, así como logros educativos y sociales (Organización Mundial de la Salud, 2017). Diversos autores en diferentes países registran la dificultad para la inserción laboral de las personas con TEA (Vidriales et al., 2018; Lewis, 2016; Dudley et al., 2015).

Específicamente, los jóvenes con TEA sin discapacidad cognitiva y sin actividades diarias programadas se encuentran en condición de vulnerabilidad, tienen mejor nivel de habilidades sociales e independencia funcional que los que presentan discapacidad cognitiva, aunque tienen menos habilidades para ser independientes que aquellos jóvenes que no presentan dificultades y pudieron seguir estudiando (Taylor et al., 2015; Taylor y Mailick, 2011).

La falta de habilidades sociales es una de las dificultades que tienen los jóvenes con TEA para encontrar o conservar un trabajo, motivo por el que el entrenamiento en habilidades sociales puede ser considerado como una necesidad (Antón et al., 2016; Cuesta et al., 2016; Neary et al., 2015).

De igual modo, debido a sus características, los jóvenes con TEA ven limitadas sus opciones de involucrarse en actividades artísticas y de recreación, lo que implica una limitación de su expresión a través del arte, convirtiéndose ésta en una de las áreas de mayor exclusión para las personas que presentan una discapacidad. Considerando estos aspectos, cabe valorar que la participación en la disciplina del teatro puede contribuir a enriquecer el repertorio conductual y social de las personas con TEA (Blanco et al., 2016; Calafat-Selma et al., 2016; Corbett, 2016; Guli et al., 2013, Kempe y Tissot, 2012).

Es conocido que el teatro como actividad artística explora aspectos de la experiencia humana adentrándose en sus pensamientos y conductas, permite abordar situaciones análogas de la vida cotidiana y contribuye al desarrollo personal, social y emocional. En su práctica se pueden experimentar diferentes relaciones con otros y descubrir las consecuencias de sus acciones de manera segura, y también permite enfrentarse a la resolución de problemas cotidianos en situaciones ficticias (Sherratt y Peter, 2002).

Existen experiencias documentadas de la utilización del teatro para el desarrollo de las habilidades sociales de personas con TEA que varían en complejidad y alcance. Tres programas estructurados, sistematizados y evaluados son The Miracle Project, The Shakespeare Heartbeat y SENSE Theatre (Corbett et al., 2011).

The Miracle Project fue creado en 2004 en California, Estados Unidos, con el fin de brindar a personas con autismo y otras discapacidades herramientas para desarrollar habilidades de comunicación, sociales, comunitarias y mejorar la autoestima, por medio de programas de teatro inclusivo y de expresión artística. Kim y Boyns (2015) señalan que este planteamiento propicia que trabajen en conjunto con jóvenes sin discapacidad. Esto posibilita el poder establecer relaciones con pares que no presentan el trastorno y que estos conozcan y entiendan mejor a los jóvenes con TEA. El trabajo se realiza con una intervención donde ambos grupos experimentan y practican su presentación, comparten sus respectivas realidades y funcionan como modelos. 
Como resultado a corto plazo, la intervención repercute en la mejora de la autoestima, comunicación, confianza y empatía de los jóvenes con TEA. A largo plazo, se aumenta la calidad de sus relaciones sociales, así como de habilidades para la vida.

En cuanto a los beneficios para otras poblaciones, se espera que los jóvenes sin condición de TEA mejoren la aceptación hacia sus pares con TEA, que los familiares vean reducido su estrés por el apoyo del grupo y que la audiencia se sensibilice hacia las personas con TEA (Kim y Boyns, 2015).

De acuerdo con Hall e Issacs (2011), el proyecto Miracle se concibe para formar una comunidad inclusiva en la que los jóvenes voluntarios aprendan sobre el autismo y los miembros de la familia estén implicados y realicen actividades como el desarrollo del vestuario, publicidad y programa de actividades. También se cuenta con actores, bailarines y cantantes voluntarios que reciben capacitación sobre el tema. El grupo desarrolla un musical original para presentar y todo se realiza desde la consideración del proceso creativo como generador de cambio de la vida de sus participantes.

The Shakespeare's Heartbeat fue creado en Londres por Hunter en 2014. En él se realizan juegos sensoriales y actuaciones para trabajar con jóvenes con TEA, e incentivan el contacto visual, el desarrollo de conciencia sensorial y la mejora del habla y el lenguaje (citado por Mehling et al., 2016). La metodología consiste en una intervención basada en la actuación dramática con preadolescentes entre los diez y los catorce años, en la que se utilizan como base, obras de Shakespeare. Se trabajan aspectos como el contacto visual, esperar turnos, el reconocimiento de emociones faciales, la imitación motora y afectiva, el diálogo pragmático, el espacio personal, la improvisación, el humor y la comunicación. Se recomienda que haya un facilitador por cada tres niños para que puedan tener atención personalizada y utilizar componentes de entrenamiento en habilidades sociales. Esta metodología permite a los participantes desarrollar trabajo colaborativo, liderazgo y capacidad de seguir el liderazgo de otros. Este método no tiene como finalidad la realización de una obra de teatro sino que se limita a utilizar las técnicas teatrales para el desarrollo de habilidades.

Fue evaluado en la Universidad de Ohio, EE.UU., con catorce participantes, y encontraron en un análisis post intervención un incremento en las medidas de habilidades sociales, comunicación y lenguaje pragmático, así como en la habilidad de reconocer emociones (Mehling et al., 2016).

El Sense Theatre (Corbett et al., 2011) está ampliamente documentado por la Universidad de Vanderbilt, EE.UU. Es un programa de teatro endocrinólogo y socioemocional desde las neurociencias, desarrollado por el equipo de la doctora Blythe Corbett de la referida universidad. Se utiliza el teatro como intervención y se realizan mediciones neuropsicológicas, biológicas y conductuales para analizar los resultados tras la intervención (Corbett, 2016; Corbett et al., 2011).

La metodología se basa en la interacción con pares, los participantes realizan juegos de teatro, role-play, improvisación, ejercicios de actuación y la presentación pública de una obra de teatro, actividades que permiten a los participantes interactuar con sus iguales y poner en práctica sus habilidades sociales. Las intervenciones con este programa han reportado resultados moderados de disminución de la activación fisiológica relacionada con la ansiedad y muestra evidentes ganancias en percepción social, funcionamiento social y habilidades adaptativas de los participantes (Corbett et al., 2014). Este tipo de intervención fue evaluada con una prueba aleatoria cuyos resultados respaldan su eficacia tanto para la mejora de las competencias sociales (Corbett et al., 2015) como para el manejo de la ansiedad (Corbett et al., 2016). 
El procedimiento consiste en que los jóvenes voluntarios reciben un entrenamiento previo a las sesiones de trabajo. Con posterioridad, cada uno es asignado a un participante del mismo sexo y edad similar. Se trabaja una primera sesión de contacto seguida de juegos de teatro guiados por pares, juego imaginativo, seguimiento de guiones, role-playing, y realización de juegos musicales. Posteriormente se inicia el montaje de la obra de teatro y la construcción de personajes, y se finaliza con sesiones de pruebas técnicas con sonido, luces y vestuario. El proceso concluye con la presentación de las dos funciones de la obra de teatro.

En Costa Rica, las posibilidades de formación, recreación y trabajo se ven limitadas para los jóvenes que presentan una situación del trastorno del espectro autista, en especial al terminar los estudios de secundaria y no tener opciones reales de inserción social, laboral o de estudios superiores. Esta inquietud, manifestada por las familias, y de forma especial por las madres de un grupo de estudiantes con esta condición, fue la base para el diseño y nacimiento de este proyecto de teatro dirigido a personas con TEA en el seno de la Universidad Estatal a Distancia.

La iniciativa nace en el año 2015 y tiene como objetivo el desarrollar y mejorar las habilidades sociales de sus participantes a través de la expresión artística, en un ambiente real de teatro. Se trabajan cuatro categorías: reconocimiento de emociones, trabajo en equipo, comunicación y autocontrol a través de técnicas de teatro. A lo largo del proyecto se ha trabajado con un grupo estable desde el año 2016 hasta el 2018. En 2018 se sumó un segundo grupo de formación básica y en 2019 se realizó una fusión entre ambos grupos. En total se han realizado cinco obras de teatro (cuatro por el grupo en estudio presentado en este artículo y uno más por un segundo grupo de formación) y se ha divulgado el proyecto por varios medios de comunicación escrita, radiofónica y televisiva (Rodríguez, 2018; Ramírez, 2017; Kcuno, 2016).

La finalidad y actividades de este trabajo tienen relación con los objetivos de la Organización de las Naciones Unidas (2016), para promover, entre otros, la educación inclusiva, los procesos de calidad y la formación a lo largo de la vida. En este sentido, (Antón, 2018) refiere que en el programa de la Agenda 2030 figuran diecisiete objetivos para transformar el mundo; entre ellos, destacamos por su relación con este trabajo el Objetivo 4, que pretende garantizar una educación inclusiva y equitativa de calidad así como promover oportunidades de aprendizaje permanente para todos (Organización de las Naciones Unidas, 2018).

\section{Metodología}

La experiencia se ha desarrollado como metodología de investigación-acción, construcción de conocimiento a través de la práctica. Su objetivo es realizar prácticas concretas para resolver problemas cotidianos e inmediatos, de manera que se puedan aportar datos que permitan tomar decisiones para programas y procesos. Este tipo de investigación busca mejorar la calidad de vida de las personas involucradas y se pretende que éstas tengan mejor conocimiento de su realidad. Es necesario que el equipo de investigación y todas las personas involucradas en el proceso estén en constante interacción. De acuerdo con Hernández et al. (2010), el proceso requiere detectar un problema, elaborar un plan para abordarlo, implementarlo, evaluarlo y finalmente, recibir retroalimentación para realizar ajustes que serán reevaluados. 


\subsection{Participantes}

Los participantes que realizaron el proceso completo desde 2016 hasta 2018 fueron ocho estudiantes con diagnóstico de TEA, cinco hombres y tres mujeres con edades entre los 12 y los 22 años, con una edad promedio de $17,14$ años ( $D E=4,27)$. Se establecieron como criterios de selección, que tuvieran un diagnóstico de TEA y que se refiriera una necesidad de desarrollo o fortalecimiento de habilidades sociales. Se desestimó la participación de menores de 12 años.

\subsection{Desarrollo de las sesiones}

Se han realizado noventa y cinco sesiones de teatro y dieciséis sesiones de tecnología. Las sesiones de teatro se planificaron como talleres semanales de hora y media todos los martes entre los meses de marzo y diciembre, durante tres años, de 2016 a 2018. El taller de teatro del primer año se diseñó para abordar cuatro objetivos básicos de actuación, denominados: actuar es jugar, actuar con verdad, situación-conflicto y resolución de situaciónconflicto. Se desarrollaron ejercicios y dinámicas y se procedió al montaje de una primera obra de teatro, titulada Querido Santa, que se presentó al finalizar el primer año. El segundo año de taller de teatro se focalizó en el montaje de dos obras, una para presentar en junio, El árbol de la vida, y otra en diciembre, Una Navidad para recordar. El tercer año se dedicó en su totalidad al montaje de su cuarta obra de teatro, esta de corte naturalista y enfocada en una problemática real como el acoso escolar, llamada Así soy yo. Todas las obras son originales, elaboradas para el grupo durante las sesiones de ensayo con aportes de los jóvenes bajo la dirección del escritor y director del teatro, así como el apoyo de la docente del grupo.

El montaje consiste en sesiones donde los participantes aprenden sus diálogos, canciones y coreografías, para practicarlo en grupo, bajo la dirección de los profesores de teatro.

En paralelo a este trabajo de técnicas teatrales y montaje de funciones, los participantes han recibido formación en tres talleres del área de tecnología en coordinación con el equipo del fab lab Kä Träre de la Universidad Estatal a Distancia. Esta coordinación permitió que los participantes tuvieran la posibilidad de recibir un taller impartido por el equipo interdisciplinar del laboratorio compuesto por profesionales en computación, diseño y ciencias de la comunicación y de psicología. Estos profesionales no tenían formación específica en educación especial, motivo por el que, antes de comenzar los talleres, recibieron capacitación para adquirir conocimientos sobre aspectos generales relacionados con el TEA.

El primer taller consistió en electrónica y luces; en él construyeron una máscara de madera pintada con luces led insertadas que utilizaron en la primera función. En el segundo, aprendieron programación básica por bloques con Scratch. Se eligió este tipo de lenguaje de programación, desarrollado por el Instituto Tecnológico de Massachusetts, por ser un recurso de aprendizaje lúdico, significativo y que propicia la interacción social. Se utiliza para trabajar con personas con necesidades específicas, ya que permite construir conocimientos de manera activa, planificar proyectos, plantearse preguntas y trabajar en resolución de problemas (López-Escribano y SánchezMontoya, 2012). Sus características, ligadas al fácil entendimiento del pensamiento computacional, han hecho que sea muy difundido actualmente en la educación de niños, adolescentes y adultos (Lifelong Kindergarden, 2018). 
Su aplicación les permitió crear historias ambientadas en el universo de la obra de teatro que estaban montando. El tercer taller consistió en la elaboración de faroles navideños con secuencias de luces programadas de manera personalizada por cada uno de los participantes a través de programación por bloques.

\subsection{Equipo de trabajo}

El proyecto fue diseñado y desarrollado por las autoras de este trabajo, como proyecto de investigación de la Cátedra de Conceptualización de la Educación Especial de la UNED. El taller fue impartido por profesionales de las artes escénicas en el contexto real de un teatro de tradición en la ciudad de San José, el Teatro La Máscara. El equipo de trabajo está compuesto por tres docentes de educación especial, una terapista de lenguaje, un comunicador y una psicóloga, así como voluntarios con experiencia en danza, música, otros actores profesionales y amateurs entre los seis y los veinte años, que participan como apoyo en las representaciones y acuden a las sesiones semanales. Hay personas del equipo que actúan en las obras de teatro mientras que otras, durante las presentaciones, se encargan del trabajo entre bastidores. Merece ser destacado que se involucró a las familias como participantes activos de todo el proceso, se implicaron como informadores del avance de sus hijos fuera del teatro, observando activamente sus progresos, interactuando con los participantes antes y después de los talleres, realizando actividades grupales con los jóvenes y asumiendo el rol de promoción del evento, distribución de entradas y recibimiento del público el día de la función.

\subsection{Recolección, sistematización y análisis de información}

Se han utilizado diversos métodos y estrategias para recabar información a lo largo del proceso, los cuales se detallan a continuación:

- Bitácora de trabajo: la docente a cargo de las clases de actuación completa cada semana una bitácora donde califica por cada participante su desempeño en las actividades realizadas.

- Bitácora de tecnología: las personas facilitadoras de los talleres de tecnología han completado registros del desempeño semanal de los participantes y de aspectos que pudieron observar relacionados con las cuatro habilidades sociales estudiadas.

- Guía de observación de sesiones: cada taller ha contado con dos observadoras no participantes quienes toman nota de las conductas manifestadas relacionadas con cada una de las categorías: expresión de emociones, comunicación, autocontrol y trabajo en equipo.

- Grupo de discusión con familias: se han realizado reuniones anuales con madres y padres para recopilar su apreciación sobre los cambios logrados por sus hijos e hijas con el paso de las sesiones. Estos grupos han sido modulados por una facilitadora y documentados por las notas tomadas por esta y observadores de la sesión.

- Observaciones familiares: a cada familia se le ha hecho entrega, una vez al año, de una guía de observación basada en el ABAS-II, en el cual recopilan la frecuencia con que se presentan algunas conductas adaptativas de los participantes en su vida cotidiana, para comparar los avances entre los distintos años. 
- Discusión del equipo investigador: en las reuniones anuales del equipo de investigación se discuten los avances de cada uno de los participantes y el grupo general, lo que se hace constar en la minuta de reunión.

Contar con las apreciaciones de las observaciones no participantes, las familias y los facilitadores de los diferentes talleres, en conjunto con las discusiones de un grupo de investigación transdisciplinario, ha permitido fortalecer la confiabilidad y validez de la información recopilada y los análisis realizados a partir de esta.

La información recolectada a través de estos instrumentos se evalúa de manera individual y luego se clasifican los avances registrados por medio de análisis de contenido a partir de las cuatro grandes categorías que se desprenden de los objetivos: emociones, comunicación, autocontrol y trabajo en equipo. Se han realizado cortes de evaluación con cada obra de teatro desde lo interno del grupo, así como evaluaciones anuales con las familias. Cada categoría se ha entendido de la siguiente manera:

- Emociones: todas las conductas en lo relacionado con expresión verbal y no verbal de emociones, así como la identificación de las emociones de otros y la interpretación de la intención de su personaje.

- Comunicación: todas las conductas de intercambio de información con otras personas. Se ha dividido en comunicación con pares, con docentes y con personas ajenas al grupo de teatro.

- Autocontrol: se han contemplado los aspectos de reacción a texturas extrañas, manejo de la ansiedad, regulación emocional, tiempos de espera y concentración, seguimiento de instrucciones y tolerancia a la frustración.

- Trabajo en equipo: se enfoca en el trabajo con otros, dividido en conductas relacionadas con apoyo a docentes y adultos, apoyo entre compañeros ajeno a la instigación o instrucción de un adulto, conductas de liderazgo, flexibilidad para trabajar con otros.

Además de estas categorías, se ha evaluado el desempeño en las actividades, clasificado en tres niveles: no lo logra, en proceso de lograrlo y lo logra. Para la presentación de resultados se utilizarán pseudónimos para resguardar la identidad de los participantes, los cuales han autorizado por medio de consentimiento informado de ellos o de sus encargados legales la utilización de los datos recopilados en el proyecto para fines de investigación.

\section{Resultados}

Este proyecto de teatro y tecnología contribuye a nutrir la investigación en el uso de técnicas teatrales para trabajar con personas con TEA, integrando además aspectos tecnológicos. En tres años de proyecto, es posible identificar resultados concretos con respecto a cada una de las categorías estudiadas, así como en el desempeño de los jóvenes dentro del taller de teatro. La información obtenida permite conocer y aplicar los alcances de este tipo de intervención y reflexionar acerca de los componentes que debe tener una siguiente etapa del proyecto en la que la metodología de trabajo se implemente con nuevos grupos. 
A continuación, se presentan los resultados por cada una de las categorías, en las que se integran los resultados obtenidos en cada uno de los espacios evaluados.

\subsection{Desempeño en los talleres}

El desempeño ha sido medido por actividad para cada estudiante semana a semana, con la valoración docente sobre si completan las actividades realizadas en clase y el montaje final de la obra de teatro. En cuanto a las actividades en clase, la más frecuente es el calentamiento; éste se realiza al inicio de casi todas las sesiones y comprende ejercicios de estiramiento y relajación. Esta actividad durante el año 2016 no logró ser completada en ningún momento por una participante; ésta mejoró en su ejecución de manera progresiva, aunque no logró desempeñar la totalidad de su papel. El resto sí lograron completarla. En el año 2018, todos han logrado realizar la actividad con éxito, en todas las sesiones. Lo más destacado sobre este avance en las actividades de calentamiento, es el hecho de que este implica un fortalecimiento de habilidades como seguir instrucciones, tolerancia a posturas y movimientos incómodos, control de la fuerza y el movimiento, regulación de la respiración y de la respuesta motora.

Los ejercicios de actuación se han variado cada año, dependiendo de la dificultad con la que el grupo puede lidiar, yendo siempre hacia actividades de mayor complejidad. En este caso, las dificultades para completar actividades están asociadas a condiciones propias de cada participante, como dificultades cognitivas para comprender las instrucciones o la abstracción en los casos que hay que utilizar la imaginación.

Sí se ha evaluado igual cada año el montaje de la obra, que ha ido aumentando el nivel de dificultad respecto al anterior, se ha incrementado la cantidad de texto de cada participante, el número de escenas en que participan, los movimientos en el escenario y la complejidad de los personajes, emociones y situaciones que deben representar. Se ha avanzado de dos primeras obras donde todos eran seres fantásticos a dos obras donde todos son seres humanos con problemas cotidianos, en especial en la cuarta obra, donde se aborda una problemática tan compleja y cercana como el acoso escolar.

Al calificar el desempeño de cada participante en el montaje de las obras se toma en cuenta que sean capaces de recordar sus textos, los pies de entrada, la posición sobre el escenario y la secuencia de las escenas. Al valorar el desempeño de los seis participantes que han participado en las cuatro obras de teatro, se encuentra que para la primera obra, solo tres lograron exitosamente el montaje mientras tres se quedaron en proceso. En el segundo año, en el que se realizaron dos montajes, cinco participantes lo lograron y solo una se quedó en proceso. Para el tercer año, los seis lograron el montaje de la obra que presentaron al final del proceso. En la figura 1, puede verse que, a pesar del grado de alcance de cada uno, en todas las ocasiones los seis participaron de la presentación final. 


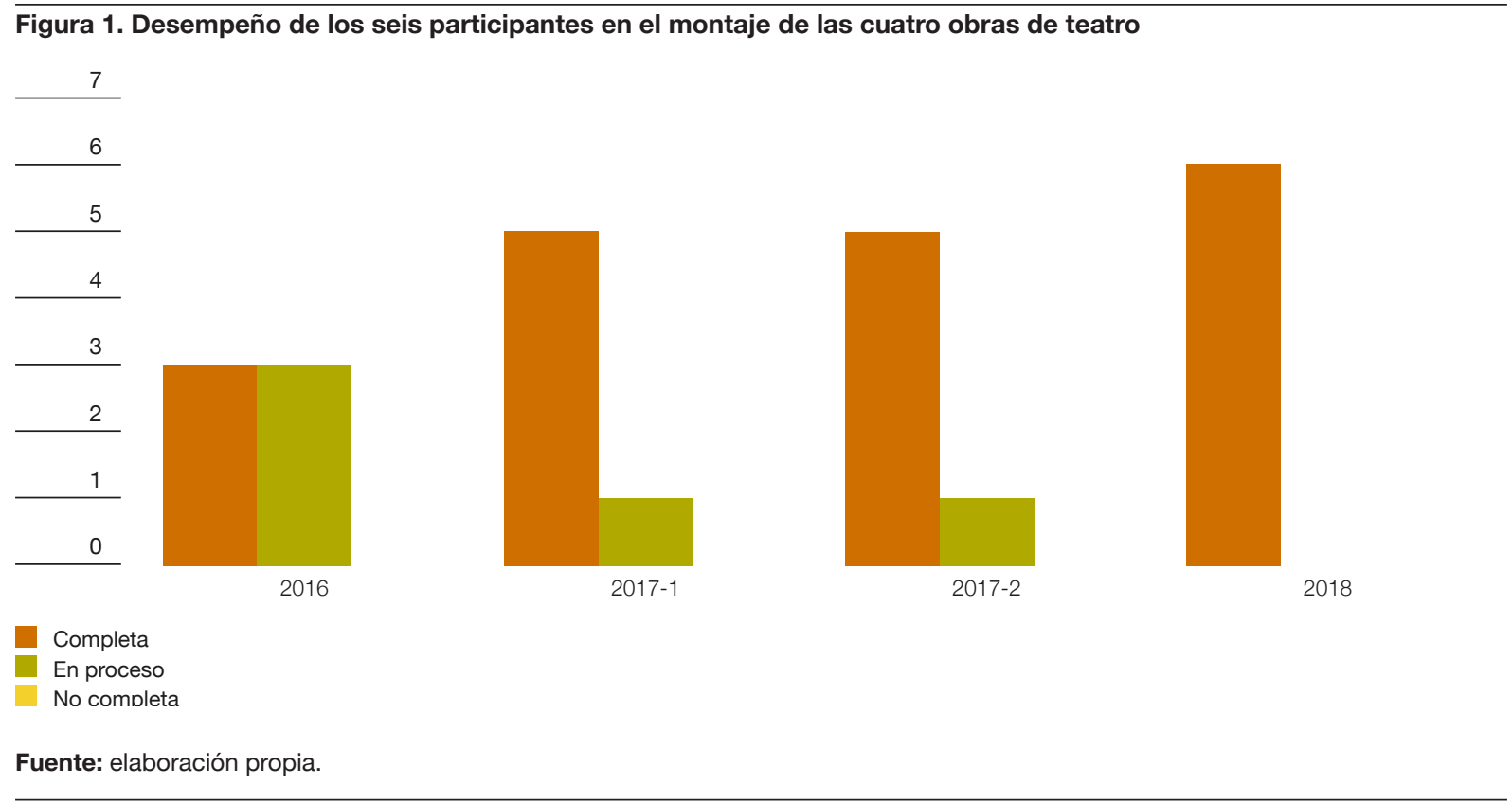

\subsection{Emociones}

Los jóvenes progresan en su capacidad de imprimir intención al personaje, esto es, transmitir las emociones que su personaje está experimentando en cada escena. En ocasiones presentan dificultades para mantener la expresión de una emoción todo el tiempo cuando representan una situación. Sin embargo, esto ha mejorado. En el análisis final de bitácoras de 2017 se refleja que dos de los ocho participantes logran mantener la emoción de su personaje cuando está en escena. Para el análisis de bitácoras de 2018 únicamente se observa, lo siguiente, para dos estudiantes:

Necesita apoyo para integrar la expresión de emociones a la actuación del personaje de manera sostenida dado que, por ejemplo, cuando se le pide mostrarse enojado lo hace un momento, sin lograr sostener la expresión a lo largo de la escena o actuación.

(Resultados de bitácoras del año 2018)

Más allá de la actuación, ha sido posible observar que expresan un amplio rango de emociones entre entusiasmo (sonrisas, aplausos), preocupación por completar su trabajo ("es que no me sale"), curiosidad (acercarse a la docente antes de iniciar la clase para ver qué van a hacer), pena (llanto o expresiones de tristeza), ansiedad (movimientos estereotipados, caminar con inquietud de un lado a otro), molestia (ceños fruncidos, puños apretados), felicidad (risas, aplausos), gusto y disgusto por las tareas que realizan. Dos madres señalan que han apreciado más habilidad en sus hijos para expresar lo que sienten. En su mayoría manifiestan entusiasmo por que sea martes y asistir a las sesiones de teatro, siendo frecuente ver que sonríen durante las actividades y que según indican las madres:

Todos los participantes (menos uno) manifiestan entusiasmo por asistir a las sesiones de teatro y sus padres pueden percibir que disfrutan el espacio, tanto por lo que dicen como por sus diferentes manifestaciones de deseo de que 
sea martes. Por ejemplo, "Gerardo nunca se había logrado aprender los días de la semana. Ahora sabe contarlos para saber cuánto falta para que sea martes."

(Notas del grupo de discusión de familias, 24 de junio de 2017)

Además de mejorar en su capacidad de expresión emocional, es destacable el desarrollo de habilidades para identificar cuando alguna persona se encuentra triste o molesta y para preocuparse por ella, aunque no puedan precisar necesariamente la emoción. Así como también, preocuparse por la ausencia de alguien. Esto ha sido posible observarlo a través de las preguntas que los jóvenes realizan directamente a la persona que identifican con malestar o al equipo facilitador.

Iván se preocupó por Jazmín y le preguntó por qué estaba "cansada" pero ella no le contestó.

(Notas de taller de programación sobre las conductas de un participante mientras una compañera está teniendo un berrinche, 17 de mayo de 2017)

Adán pregunta por Marta, porque ésta no había llegado.

(Observación de sesión, pregunta al inicio de sesión, 6 de marzo de 2018)

\subsection{Comunicación}

Se ha percibido un aumento de la frecuencia de interacciones entre los participantes en los ensayos y los recesos, en especial cuando ellos proponen un tema de conversación de su interés. De acuerdo con (Antón et al., 2016), las estrategias de aprendizaje para el desarrollo de las competencias comunicativas de los jóvenes en riesgo es un reto que merece ser considerado y abordado desde un enfoque multidisciplinar y en diferentes contextos. En este caso, se ha observado que la metodología seguida ha contribuido, en general, a que se produzca mayor interacción con sus pares. Comparten con el grupo temas personales, comentan si van a viajar, si tienen alguna actividad importante o sobre sus gustos y preferencias. Un avance no planificado en la investigación pero que surgió del propio grupo fue la creación de un grupo de WhatsApp, con el que se comunican durante la semana.

En general, en 2018 el análisis de observaciones de todos los participantes indica fortalezas y progresos en el área de comunicación con sus compañeros:

Adán: tiene iniciativa para comenzar conversaciones con propios y extraños, comparte temas personales.

Gerardo: muestra iniciativa para saludar y preguntar por los demás.

Iván: busca en todas las sesiones conversar con otros por medio de preguntas o historias, incluso chistes. Ha diversificado los temas de interacción. Disminuyó la frecuencia en que propone a los otros juegos e interacciones estereotipadas como repetir números.

León: intenta hablar con compañeros sobre temas de interés propio.

Marta: responde a los saludos de sus compañeros en lugar de ignorarlos.

Sonia: habla con sus compañeros y los escucha cuando se acercan a ella.

(Resultados de bitácoras del año 2018)

1. Por respeto a la intimidad, los nombres que figuran en las anotaciones, no corresponden a los participantes, se han adjudicado nombres ficticios. Sí es real la información recopilada y registrada. 
Ha aumentado la frecuencia en que los componentes del grupo se dirigen a los equipos facilitadores de los talleres tanto para hacer consultas como para comentar temas personales. Han aprendido a verbalizar sus quejas en situaciones en las que algo no les parece bien en la clase o acerca de sus compañeros con comentarios como “¿cuándo me toca a mí?”, “¿por qué no lo hacemos diferente?” o señalando ejecuciones o acciones incorrectas de sus compañeros de manera no verbal. De hecho, han desarrollado confianza para hacer indicaciones o corregirse entre ellos, al equipo investigador o incluso a los profesores de teatro.

León: [a una profesora que se equivoca con la línea que debe decir en la obra] es que tienes que estudiarte el texto.

(Bitácoras del año 2017)

Las familias refieren mayor seguridad por parte de sus hijos para interactuar con otras personas, como lo ilustra la madre de Sonia.

A ella le cuesta hablar con chicas de su edad porque tienen intereses diferentes. Pero sí tiene más libertad ahora para hablar con las personas. Ella habla muy bajo, pero ahora con quienes tiene familiaridad como en el teatro, habla más fuerte. El teatro le ha ayudado a pararse de una manera diferente y con la timidez.

(Notas del grupo de discusión de familias, 24 de junio de 2017) ${ }^{2}$

Otras familias expresan que han notado avances similares. Esto se corresponde por la iniciativa observada en los participantes para acercarse a otras personas cuando llegan al teatro, pero que no son del grupo, como cuando han llegado periodistas o actores que tienen otras funciones en el teatro. Se acercan a ellos y se presentan para después preguntarles quiénes son ellos y qué hacen ahí.

En general, se producen mejoras en la comunicación asertiva y la funcionalidad de la comunicación, pero sobre todo en la seguridad que muestran los participantes para interactuar con otros.

\subsection{Autocontrol}

Las mejoras en autocontrol han sido notorias en diversos ámbitos: espera de turno, seguimiento de instrucciones, control de la frustración, periodos de atención, tolerancia de texturas, manejo de la ansiedad y regulación en la demostración de emociones.

Se han producido mejoras en tolerar los tiempos de espera para ensayar y la capacidad de esperar turnos, aspecto necesario para tener conversaciones grupales ordenadas y coherentes y para participar en escena en el momento que les corresponde. Esto se visualiza cuando son infrecuentes las interrupciones durante el desarrollo de las sesiones, se ha reducido la frecuencia de situaciones en las que un participante empieza a hablar de otro tema o diga las líneas de su intervención, antes del momento en que le corresponde en los ensayos. Además, todos esperan sin problema su turno, sin salir a escena durante las funciones, hasta el punto de que ha desaparecido el intentar asomarse para ver lo que sucede fuera mientras los otros actúan.

Ha aumentado la duración de los periodos de atención. Se empezó a trabajar en sesiones de una hora, después se amplió a hora y media con receso, hasta llegar a trabajar el último año dos horas seguidas, sin receso, en las

2. En Costa Rica, se entiende por "pararse", en este caso, la postura corporal de estar de pie. 
últimas sesiones de ensayo. A esto se une la concentración en la tarea y en especial en las presentaciones teatrales, para estar al tanto de lo que ocurre en escena y saber cuándo les corresponde participar.

El trabajo con los ejercicios de actuación, el calentamiento y los talleres con tecnología han favorecido todo lo relacionado con la capacidad de seguir instrucciones, atención y concentración en las tareas, así como la tolerancia a la frustración cuando algo no ha funcionado en un primer intento.

Además, se ha trabajado la tolerancia de texturas con vestuario y maquillaje que, en algunos casos, les producía incomodidad o molestia y que poco a poco lo han aprendido a tolerar como parte de la actuación sin desencadenar negativas o berrinches.

Un tema recurrente durante los tres años, ha sido el manejo de la ansiedad, tanto por salir a escena como por aspectos de trabajo cotidiano en el teatro. Ha disminuido la frecuencia en que aparecen en la mayoría conductas estereotipadas o autoestimulantes, aunque todavía es posible apreciarlas en algunas sesiones. Ninguno depende ya de objetos de seguridad para salir a escena (como una joven que necesitaba llevar siempre puesto su bolso) y todos participan de los montajes y presentaciones sin que los cambios que pueda haber por razones de tiempo, tipo de escenario o utilería resulten en una crisis o dificultad para desempeñarse.

Otro logro alcanzado, como resultado de trabajar el control y la regulación de la demostración de emociones, ha sido la mejora de las expresiones de alegría o enojo, disminuyendo la frecuencia de gritos o berrinches. También se ha trabajado en adoptar maneras apropiadas para demostrar afecto hacia sus compañeros y profesores, sin invadir el espacio personal.

Varios de estos avances se vienen apreciando desde el año 2017, como reflejan las siguientes reflexiones del grupo de investigación:

Los participantes se muestran empoderados en el escenario, son capaces de improvisar y tolerar que algo no salga exactamente como se había ensayado, así como los constantes cambios en el texto. Aguantan sesiones de ensayo de hora y media a dos horas sin pedir recesos o, si lo piden, es con poca frecuencia.

(Análisis del equipo de investigación, 2017)

Algunas notas de las observaciones de 2018 que evidencian estos progresos son las siguientes:

Adán: desarrolla bien las tareas cuando se concentra en ellas.

Gerardo: logra esperar turnos con mayor tranquilidad. Muestra mayor control para participar de las actividades controlando su fuerza y movimientos al realizar ejercicios con los otros.

Iván: aumentó la concentración durante las escenas, recuerda sus diálogos y pies de entrada con menos apoyo. Marta: tiene más control en escena, no se sale de su papel ni rompe la cuarta pared para saludar al público.

Sonia: se automotiva con expresiones como "yo puedo" o "lo hice bien". Realiza los intentos que sean necesarios para lograr algo.

(Resultados de bitácoras del año 2018) 


\subsection{Trabajo en equipo}

El trabajo en equipo es la categoría que ha requerido más tiempo para observar y registrar resultados. Debido a que en los dos primeros montajes fue necesario llevar un proceso más personalizado, en el que cada uno comenzaba por aprender sus textos y movimientos, todos mostraban preferencia por el trabajo individual. Sin embargo, incluso entonces, todos los participantes coincidían en escena y se desarrollaron colaboraciones puntuales entre ellos. Algunos indican a otros dónde deben situarse en el escenario, recordarles líneas o guiarles para entrar o salir de escena.

Para la tercera obra, sin embargo, el montaje requería que hicieran escenas en subgrupos donde no necesariamente había algún voluntario profesional con ellos, de manera que empezaron a desarrollar estrategias para sacar sus escenas adelante. Fue posible observarlos ensayar solos entre ellos sin que mediara indicación de la facilitadora, se les pudo escuchar animarse e incluso resolver discusiones personales antes de salir a escena.

León y Sonia ensayaban constantemente tras escena juntos. León ayudaba a Sonia a repetir sus diálogos y sobre el escenario también la apoyaba recordándole líneas.

Adán tuvo que desarrollar una mayor tolerancia para el trabajo en equipo dado que Gerardo tiene mayores dificultades para montar una escena y Roberto [voluntario] era nuevo en el grupo. Los tres practicaban también sus escenas mientras no estaban sobre el escenario.

Los participantes en general se mostraron dispuestos y capaces de improvisar sobre el escenario, resolver y colaborar con aspectos técnicos como traer y colocar utilería.

(Análisis del equipo de investigación, 2017)

También se presentan conductas de apoyo a las facilitadoras, como ayudarles a localizar objetos, colocar escenografía o buscar las canciones en la computadora. Fue posible ver al grupo improvisar y resolver escenas juntos, así como una mayor colaboración entre todos para resolver aspectos técnicos de montaje y utilería.

Adán: colaboró constantemente con recordar las líneas, salidas y movimientos de todos los participantes, de lo que guardaba muy buena memoria. Cuando se le decía que no debía estar afuera, dijo que él era quien estaba ayudando a la profesora.

(Observaciones de sesiones, 2017)

En el 2018 fue posible ver a un grupo pendiente no solo de sus escenas y líneas sino de las de todo el grupo, desarrollando conductas de apoyo a los demás como indicarles cuándo les corresponde salir o felicitarlos cuando salen de escena porque lo hicieron bien.

Las familias aprecian que existe un sentido de pertenencia y de integración entre ellos como grupo de trabajo, pues señalan que se percibe que "se cuidan, se entienden, se quieren" y han encontrado en el grupo un lugar donde son aceptados y les gusta estar. 


\subsection{Otros resultados de interés}

Fruto de la actividad formativa ha sido el poder presentar cuatro obras completas, siete presentaciones públicas (una de la primera obra, dos de la segunda, una de la tercera y tres de la cuarta), diez apariciones del proyecto en radio, tres en televisión y cinco en prensa escrita (Kcuno, 2018; Kcuno, 2016; Rodríguez, 2018; Ramírez, 2017; Sanabria, 2019). Se ha presentado en varios eventos académicos nacionales como las Jornadas Psicorecreactivas 2016 de la Universidad de Costa Rica, el Congreso Internacional de Investigación sobre Discapacidad 2016, el Congreso Internacional de Innovación y Tecnología en la Educación a Distancia de 2017, el I Congreso Nacional de Organizaciones de Personas con Autismo en 2019 y las V Jornadas de investigación en artes de la Universidad de Costa Rica en 2019. Además, en España tuvo una presentación en el XV Congreso Internacional y XXXV Jornadas de Universidades y Educación Inclusiva: "Liderando investigación y prácticas inclusivas" (Madriz et al., 2018).

También se realizó en 2017 una campaña de sensibilización y concienciación sobre los estereotipos y las etiquetas relacionadas con el autismo, promovida por la UNED y en la que participaron todos los involucrados en el proyecto. Esta se realizó a través de un vídeo producido por la UNED y divulgado en redes sociales durante el día 2 de abril, dedicado a la concienciación sobre el autismo. Al siguiente año, también para el mes de abril, se realizó un vídeo con la canción "Coincidir", cantada por los participantes, que se divulgó por el mismo medio durante el mes de abril.

La valoración positiva por parte de todos los involucrados en los dos primeros años de proyecto llevó a que en el año 2018 se ampliara la iniciativa a un segundo grupo de formación; en él se han aplicado las prácticas que han sido positivas y exitosas de la primera experiencia y, a la vez, se ha ampliado el rango de trabajo a cualquier población que necesita apoyo para el desarrollo de habilidades sociales. Los resultados de esta segunda etapa están en proceso de análisis pero, según las referencias de los familiares, también están siendo positivos.

Con la ampliación del grupo, tanto en número de participantes como en los perfiles de estos, llegó también la consolidación del mismo, el cual al inicio del proyecto no tenía nombre propio pero fue bautizado en 2017 bajo la denominación de Grupo de teatro Rompecabezas. En él están incluidas todas las personas participantes en el taller, con y sin discapacidad, profesionales, personas voluntarias y jóvenes que se inscribieron en el proyecto con un diagnóstico de TEA o con diversas necesidades, debidas a otra condición. Se ha formado un grupo que se perfila como una compañía de teatro inclusivo que, en la actualidad, cuenta como repertorio con las obras de teatro realizadas durante el desarrollo del proyecto. Todos los participantes se congregan bajo el nombre del grupo, que tiene además su propio logo y, desde el año 2019, su propia página de Facebook para la divulgación de su trabajo (Grupo de teatro Rompecabezas, 2019).

\section{Discusión y conclusiones}

El proyecto The Miracle Project tuvo comienzo en 2004 en California, Shakespeare Heartbeat en 2011 en Inglaterra (Mehling et al., 2016) y SENSE Theatre en 2010 (Corbett et al., 2011) en Vanderbilt. La trayectoria y documentación de estos proyectos los convierten en referentes importantes en el tema y de manera especial para el proyecto que presentamos, por sus numerosos paralelismos en los aspectos abordados en sus objetivos. 
La formulación del proyecto, diseño y desarrollo se realiza en el seno de la carrera de Educación Especial de la UNED de Costa Rica desde la filosofía inclusiva que busca la participación y equidad para todos.

Esta propuesta tiene numerosos paralelismos con los programas referenciados, pero a su vez tiene características particulares propias como la metodología de investigación que considera los puntos de vista de todos los participantes (equipo de investigación, profesionales, voluntarios, familias, participantes) que le brinda una identidad innovadora, por ser el primero documentado en Latinoamérica de estas características y extensión.

La metodología aplicada durante las sesiones de ensayo y representación teatral ha incluido dinámicas de juego, coordinación y desarrollo de habilidades de actuación, trabajar postura, proyección de la voz, expresión de emociones y escucha, entre otras. Durante el montaje de las puestas en escena, se realizan ensayos semanales y se trabaja el montaje de escenas conjuntas, que incluyen actividades como baile, canto y actuación.

De acuerdo con los resultados, el trabajo desarrollado dentro del teatro ha permitido el empoderamiento de los jóvenes con el espacio y favorecido que se sientan seguros en ese entorno. Se han movilizado con independencia y libertad por los vestidores, escenarios secundarios, recibidor, oficinas, butacas y escenario principal, llegando a compartir espacio, en ocasiones, con otros actores del teatro.

Los tres años de experiencia de este proyecto, con un mismo grupo de formación, han hecho posible la inmersión en la realidad de estos jóvenes, crear y evaluar posibles soluciones a sus situaciones a través del teatro y avances específicos en los participantes en cuanto a la expresión de emociones, la comunicación, el autocontrol y el trabajo en equipo. La integración de tecnología en el proceso y otorgar a los participantes el ser parte de la responsabilidad de la escenografía del montaje son características que también dotan de particularidad a este proyecto. La tecnología, como el arte, suele ser un ámbito que no acostumbra estar abierto a que personas con TEA puedan experimentar y aprender fuera de un contexto de educación especial.

Resulta vital que el grupo esté ligado a un entorno real como es el teatro La Máscara, que tiene 35 años de trayectoria y gran reconocimiento nacional y que es independiente de la universidad en la que nace el proyecto. De esta manera, el grupo trasciende el proyecto de investigación en sí para llegar a formar parte de una academia de teatro, desde donde ha podido evolucionar hasta formar su propia compañía de actores, para dotarlo de continuidad con el montaje y presentación de obras de teatro más allá del quehacer académico.

En el proceso también han participado jóvenes sin ningún tipo de discapacidad como parte del elenco de presentaciones, lo que ha permitido una interacción de pares que ha sido enriquecedora. No se ha recurrido a la presencia de pares sin discapacidad para que actúen de mediadores o modelos sino que todos han aprendido en conjunto sobre actuación y han desarrollado las puestas en escena en un plano de igualdad e inclusión.

Los criterios de inclusión de este proyecto, que admiten la entrada de adultos hasta los veinticuatro años, brinda opciones para jóvenes que ya no continúan dentro del sistema educativo formal y encuentran en el taller de teatro una oportunidad de interacción social y formación. Es un periodo especialmente delicado pues en su mayoría han sido separados de sus compañeros de educación secundaria, quienes se encuentran por lo general cursando estudios universitarios o incorporándose al mercado laboral, espacios a los que muchos jóvenes con TEA no tienen posibilidades de acceso. 
Los logros alcanzados durante estos tres años, con el progresivo avance de las habilidades de actuación de los participantes, permiten visualizar una consolidación de las artes dramáticas como verdaderas opciones laborales y recreativas para jóvenes con TEA que se encuentran, al igual que en otros países, en posiciones vulnerables, al no lograr acceder ni a estudios superiores ni a trabajos remunerados estables cuando se acercan al momento de dejar el sistema educativo.

Gracias a estos resultados surge durante el desarrollo del proyecto la idea del teatro como un fin y no solo como una herramienta para el desarrollo de habilidades sociales. Más allá de una técnica para que los jóvenes aprendan a comunicarse mejor, el teatro se ha convertido para el grupo en una opción de vocación, trabajo y oportunidades, tanto laborales como de esparcimiento y crecimiento personal. 
American Psychiatric Association (2014): Guía de consulta de los criterios diagnósticos del DSM-5. Washington. London: American Psychiatric Publishing.

Antón, P. (2018): Red Openergy: experiencias formativas e investigadoras para el diseño instruccional accesible. Education in the Knowledge Society, 19 (4): 31-51.

Antón, P. et al. (2016): Neurodidáctica y estrategias de aprendizaje para el desarrollo de las competencias comunicativas de los niños y niñas con riesgo biológico y/o social. Revista de Educación Inclusiva, 9 (1): $43-53$.

Blanco, A. et al. (2016): "El teatro como herramienta socializadora para personas con Asperger”. Revista Española de Educación y Psicopedagogía, 27: 116-125.

Calafat-Selma, M. et al. (2016): "El teatro como herramienta de intervención en alumnos con trastorno del espectro autista y discapacidad intelectual”. Revista de Educación Inclusiva, 9: 95-108.

Corbett, B. (2016): "Images of Healing and Learning. Autism, Art and Accessibility to Theater". AMA Journal of Ethics, 18 (12): 1232-1240.

Corbett, B. et al. (2016): "Changes in anxiety following a randomized control trial of a theatre-based intervention for youth with autism spectrum disorder". Autism, 3: 333-343.

Corbett, B. et al. (2015): "Improvement in social competence using a randomized trial of a theatre intervention for children with autism spectrum disorder". Journal of Autism and Development Disorders, 46 (2): 658-672.

Corbett, B. et al. (2014): "Peer-mediated theatrical engagement for improving reciprocal social interaction in autism spectrum disorder". Frontiers in pediatrics, 2: 1-9.

Corbett, B. et al. (2011): "Brief report: theatre as therapy for children with autism spectrum disorder". Journal of Autism and Development Disorder, 41: 505-511.

Cuesta, J. et al. (2016): "Trastorno del espectro del autismo: intervención educativa y formación a lo largo de la vida”. Psychology, Society \& Education, 8: 159-172.

Dudley, C. et al. (2015): "What do we know about improving employment outcomes for individuals with autism spectrum disorder?". The School of Public Policy SPP Research Papers, 8: 1-35.

Grupo de teatro Rompecabezas (2019): Grupo de teatro Rompecabezas (en línea). <https://readymag.com/UNEDInvestigaCR/GrupodeTeatroRompecabezas/>, acceso 2 de julio de 2017.

Guli, L. et al. (2013): "Social Competence Intervention Program (SCIP): 'A pilot study of creative drama program for youth with social difficulties'”. The Arts in Psychotherapy, 40: 37-44.

Hall, E. e Isaacs, D. (2011): Seven Keys to Unlock Autism: making Miracles in the Classroom. California: Jossey Bass.

Hernández, R. et al. (2010): Metodología de la investigación. México: McGrawHill.

Hunter, K. (2014): The method (en línea). <http://www.shakespearesheartbeat.com/the-method/>, acceso 12 de septiembre de 2017.

Kempe, A. y Tissot, C. (2012): "The use of drama to teach social skills in a special school setting for students with autism”. Support for Learning, British Journal of Learning Support, 27 (3): 97-102. 
Kim, A. y Boyns, D. (2015): Joining the spectrum: an interdisciplinary inquiry into theatre as an intervention for autism diagnosed teens (en línea). <https://www.arts.gov/sites/default/files/Research-Art-Works-CSUNorthridge. pdf>, acceso 12 de septiembre de 2017.

Kcuno, R. (2018): Querido Santa: una obra teatral que combina arte, destrezas y emociones (en línea). <https:// www.uned.ac.cr/acontecer/a-diario/gestion-universitaria/2783-querido-santa-una-obra-teatral-que-combinaarte-destrezas-y-emociones>, acceso 12 de abril de 2018.

Kcuno, R. (2016): Personas con espectro autista generan sinergia creativa mediante el teatro (en línea). <https:// www.uned.ac.cr/acontecer/a-diario/juncos/39-a-diario-/educacion/3276-personas-con-espectro-autista-generan-sinergia-creativa-mediante-el-teatro>, acceso 12 de abril de 2018.

Lewis, D. (2016): Best practices for facilitating independent living, employment and higher education for emerging adults with autism [tesis para optar al Grado de Doctor de Filosofía en Psicología]. The Chicago School of Professional Psychology.

Lifelong Kindergarden (2018): Acerca de Scratch (en línea). <https://scratch.mit.edu/about/>, acceso 26 de junio de 2018.

López-Escribano, C. y Sánchez-Montoya, R. (2012): "Scratch y necesidades educativas especiales: programación para todos". Revista de educación a distancia, 34: 1-14.

Madriz, L. et al. (2018): "Desarrollo de habilidades sociales en jóvenes con Trastorno del Espectro Autista (TEA) por medio del teatro", en León, M.J. y Sola, T. (coords.): Liderando investigación y prácticas inclusivas. Granada: Universidad de Granada.

Martos-Pérez, J. y Llorente-Comí, M. (2013): "Tratamiento de los trastornos del espectro autista entre la comprensión y la práctica basada en la evidencia”. Revista de Neurología, 27 (1): S185-S191.

Mehling, M. et al. (2016): "Shakespeare and autism: an exploratory evaluation of the Hunter Heartbeat Method". Research and practice in intellectual and developmental disabilities, 4 (2): 107-120.

Neary, P. et al. (2015): "Post-school needs of young people with high-functioning Autism Spectrum Disorder." Research in Autism Spectrum Disorders, 18: 1-11.

Organización de las Naciones Unidas (2016): La Asamblea General adopta la Agenda 2030 para el Desarrollo Sostenible (en línea). <https://www.un.org/sustainabledevelopment/es/2015/09/la-asamblea-general-adoptala-agenda-2030-para-el-desarrollo-sostenible/>, acceso 28 de julio de 2019.

Organización de las Naciones Unidas (2018): Objetivos de Desarrollo Sostenible (en línea). <https://www.un.org/ sustainabledevelopment/es/> acceso 28 de julio de 2019.

Organización Mundial de la Salud (2013): Medidas integrales y ordenadas para gestionar los trastornos del espectro autista (en línea). <http://apps.who.int/gb/ebwha/pdf_files/EB133/B133_4-sp.pdf>, acceso 29 de mayo de 2018.

Organización Mundial de la Salud (2017): Trastornos del espectro autista (en línea). <http://www.who.int/es/newsroom/fact-sheets/detail/autism-spectrum-disorders>, acceso 18 de mayo de 2018.

Ramírez, K. (2017): Jóvenes con trastornos del espectro autista presentarán segunda obra de teatro (en línea). $<$ https://www.uned.ac.cr/acontecer/a-diario/gestion-universitaria/2920-jovenes-con-trastornos-del-espectroautista-presentaran-segunda-obra-de-teatro>, acceso 22 de mayo de 2018. 
Riesgo, R. et al. (2013): "Evolución de los niños con retraso del desarrollo y conductas del espectro autista". Medicina (Buenos Aires), 73: 16-19.

Rodríguez, I. (2018): Teatro es aliado para mejorar destrezas de jóvenes con autismo (en línea). <https://www. nacion.com/ciencia/salud/teatro-es-aliado-para-mejorar-destrezas-de-jovenes/5FX34XYMU5FALPEVP42LLH CL2Q/story/>, acceso 22 de mayo de 2018.

Sanabria, M. (2019): Detección e intervención temprana es vital para personas con TEA (en línea). <https://adiariocr.com/educacion/deteccion-e-intervencion-temprana-es-vital-para-personas-con-tea/>, acceso 22 de mayo de 2019.

Scratch (2018): Acerca de Scratch (en línea). <https://scratch.mit.edu/about>, acceso 10 de mayo de 2018.

Sherratt, D. y Peter, M. (2002): Developing play and drama in children with autistic spectrum disorders. Londres: David Fulton Publishers.

Vidriales, R. et al. (2018): Empleo y trastornos del espectro del autismo. Un potencial por descubrir. Madrid: Autismo España.

Taylor, J. y Mailick, M. (2011): "Employment and post-secondary educational activities for young adults with autism spectrum disorders during the transition to adulthood". Journal of Autism and Development Disorders, 41: 566-574.

Taylor, J. et al. (2015): "Longitudinal patterns of employment and postsecondary education for adults with autism and average-range IQ". Autism, 19: 785-793.

The Miracle Project (2019): The Miracle Project (en línea). <http://www.themiracleproject.org>, acceso 12 de septiembre de 2017. 